Draft Version OCtober 18, 2021

Typeset using LATEX default style in AASTeX63

\title{
Exploring the stellar rotation of early-type stars in the LAMOST Medium-Resolution Survey. II. Statistics
}

\author{
Weijia Sun, ${ }^{1,2}$ XiaO-Wei Duan, ${ }^{1,2}$ Licai Deng, ${ }^{2,3,4}$ and Richard De Grijs ${ }^{5,6}$ \\ ${ }^{1}$ Kavli Institute for Astronomy \& Astrophysics and Department of Astronomy, Peking University, Yi He Yuan Lu 5, Hai Dian District, \\ Beijing 100871, China \\ ${ }^{2}$ Key Laboratory for Optical Astronomy, National Astronomical Observatories, Chinese Academy of Sciences, 20A Datun Road, \\ Chaoyang District, Beijing 100012, China \\ ${ }^{3}$ School of Astronomy and Space Science, University of the Chinese Academy of Sciences, Huairou 101408, China \\ ${ }^{4}$ Department of Astronomy, China West Normal University, Nanchong 637002, China \\ ${ }^{5}$ Department of Physics and Astronomy, Macquarie University, Balaclava Road, Sydney, NSW 2109, Australia \\ ${ }^{6}$ Research Centre for Astronomy, Astrophysics and Astrophotonics, Macquarie University, Balaclava Road, Sydney, NSW 2109, Australia
}

\begin{abstract}
Angular momentum is a key property regulating star formation and evolution. However, the physics driving the distribution of the stellar rotation rates of early-type main-sequence stars is as yet poorly understood. Using our catalog of 40,034 early-type stars with homogeneous $v \sin i$ parameters, we review the statistical properties of their stellar rotation rates. We discuss the importance of possible contaminants, including binaries and chemically peculiar stars. Upon correction for projection effects and rectification of the error distribution, we derive the distributions of our sample's equatorial rotation velocities, which show a clear dependence on stellar mass. Stars with masses less than $2.5 \mathrm{M}_{\odot}$ exhibit a unimodal distribution, with the peak velocity ratio increasing as stellar mass increases. A bimodal rotation distribution, composed of two branches of slowly and rapidly rotating stars, emerges for more massive stars $\left(M>2.5 \mathrm{M}_{\odot}\right)$. For stars more massive than $3.0 \mathrm{M}_{\odot}$, the gap between the bifurcated branches becomes prominent. For the first time, we find that metal-poor $([\mathrm{M} / \mathrm{H}]<-0.2$ dex $)$ stars only exhibit a single branch of slow rotators, while metal-rich $([\mathrm{M} / \mathrm{H}]>0.2 \mathrm{dex})$ stars clearly show two branches. The difference could be attributed to unexpectedly high spin-down rates and/or in part strong magnetic fields in the metal-poor subsample.
\end{abstract}

Keywords: Stellar rotation (1629), Astronomy data analysis (1858), Early-type stars (430), Stellar properties (1624), Stellar evolution (1599)

\section{INTRODUCTION}

Stellar rotation profoundly affects almost every aspect of stellar evolution (Maeder \& Meynet 2000). It plays a crucial role in dynamo-driven magnetic activity (Spruit 2002), stellar winds (Matt \& Pudritz 2005), surface abundances (Preston 1974), chemical yields (Prantzos et al. 2018), internal structure (Aerts et al. 2019), and external structure (Rivinius et al. 2013). Consequently, understanding stellar rotation is essential for our understanding of the origin and evolution of stellar angular momentum. However, the connection between models and observations, although much improved in the last few decades, still suffers from several shortcomings.

From a theoretical perspective, stellar rotation on the main sequence (MS) is the result of the long-term evolution of angular momentum loss and redistribution, regulated by intricate processes early in the star formation phases, e.g. the fragmentation of rotating clouds (Boss \& Bodenheimer 1979), magnetic braking (Mouschovias \& Morton 1985), bipolar overflows (Pudritz 1985), and magnetic locking with the accretion disk (Lin et al. 2011). Once the initial rotation rate at the zero-age MS (ZAMS) has been established, the rotation evolution can be described by a stellar rotation model that encompasses the physical prescriptions of meridional circulation, angular momentum transport, and (radiative, supra-Eddington, and mechanical) mass loss (Ekström et al. 2012). From an observational perspective, the observed stellar rotation rates are usually entangled with projection effects, which further complicates the use of observed rotation rates as benchmark for stellar rotation models. 
The distribution of stellar rotation rates can serve as a crucial probe of the formation and evolution processes of stellar populations. A sharp decline in rotation velocities of MS stars is found around $1.2 \mathrm{M}_{\odot}$, with more massive stars having mean rotation velocities of hundreds of $\mathrm{km} \mathrm{s}^{-1}$, while lower-mass stars have velocities on the order of a few $\mathrm{km} \mathrm{s}^{-1}$ (Kraft 1970). This is mainly so, because more massive stars lack deep convective envelopes or strong magnetic fields that slow down stellar rotation. Many studies (e.g., Abt \& Morrell 1995; Penny 1996; Huang \& Gies 2006; Huang et al. 2010) have been dedicated to deconvolve the observational distribution and derive a true rotational distribution by means of large population statistics. A bimodal distribution of rotation rates is observed among young solar-mass stars in the Orion nebula (Attridge \& Herbst 1992; Stassun et al. 1999; Barnes 2003). The origin of such a bimodality is not yet fully understood, but it is thought to be a manifestation of star-disk interactions during pre-MS evolution (Bouvier et al. 1993).

A similar bimodal distribution is also observed among massive stars $\left(M \gtrsim 2.5 \mathrm{M}_{\odot}\right)$, although the underlying mechanism might not be the same. Abt \& Morrell (1995) found a bimodal distribution of rotation velocities among A-type MS stars in the field, where the slowly rotating population was mainly ascribed to chemically peculiar (CP) stars. A large-scale study by Zorec \& Royer (2012), who reported $v \sin i$ measurements for 2014 B6- to F2-type field stars, found a substantial difference between the rotation distributions of stars with masses below and above $2.5 \mathrm{M}_{\odot}$. The less massive population exhibits a unimodal profile, while the more massive population is bimodal. They also revealed the complex behavior of stellar rotation, with strong acceleration in the first one-third of the MS evolutionary phase, followed by a mild spin-down. This calls for more efforts to improve our understanding of stellar rotation, in terms of both theory and observations.

Significant progress in stellar rotation statistics is now achievable with multi-object spectrographs acquiring data for large numbers of stars. Large spectroscopic surveys such as the William Herschel Telescope Enhanced Area Velocity Explorer (WEAVE, Dalton et al. 2012), the Galactic Archaeology with High Efficiency and Resolution Multi-Element Spectrograph (GALAH, Buder et al. 2021), the Radial Velocity Experiment (RAVE, Kunder et al. 2017), the Apache Point Observatory Galactic Evolution Experiment (APOGEE, Majewski et al. 2017), and the Large Sky Area MultiObject Fiber Spectroscopic Telescope (LAMOST, Cui et al. 2012; Liu et al. 2020) will continue to provide millions of stellar spectra and, hence, allow to unveil an unprecedentedly detailed view of stellar features. In Paper I (Sun et al. 2021), we compiled a catalog containing more than 40,000 stars with effective temperatures of $7000 \mathrm{~K} \leq T_{\text {eff }} \leq 14,500 \mathrm{~K}$ from LAMOST data release (DR) 7 medium-resolution spectra. With such a large and homogeneous sample, we aim to review the rotation distribution of early-type stars as function of their masses, ages, and metallicities.

This article is organized as follows. In Section 2, we analyze the uncertainties in $v \sin i$ in more detail. The effects of possible contamination and its influence on the derived rotation rates are discussed in Section 3 . Section 4 discusses our statistical approach to derive the true rotational velocity distribution and we further apply this method to the rotation rate distribution in Section 5. We also discuss the dependence of the stellar rotation rates on metallicity, in Section 6. Finally, our conclusions are summarized in Section 7.

\section{DATA}

Our stellar sample was defined in Paper I, where we used a data-driven method to estimate the stellar parameters and abundances ('stellar labels') of early-type candidates selected from the $\mathrm{H} \alpha$ and $\mathrm{Mg}$ I $b$ line indices provided by LAMOST DR7 medium-resolution spectra. Having adopted a selection based mainly on effective temperatures $\left(7000 \mathrm{~K} \leq T_{\text {eff }} \leq 14,500 \mathrm{~K}\right)$ and data quality, we derived a final catalog composed of 40,034 stars. The precisions of our estimates are $\sim 75 \mathrm{~K}, 0.06 \mathrm{dex}, 0.05 \mathrm{dex}$, and $\sim 3.5 \mathrm{~km} \mathrm{~s}^{-1}$ for $T_{\text {eff }}, \log g,[\mathrm{M} / \mathrm{H}]$, and $v$ sin $i$, respectively, for signal-to-noise ratios, $\mathrm{SNR}>60$. However, this only represents the average behavior of our estimates; the actual performance may vary (Sun et al. 2021). As regards the observational uncertainties, they may originate from the line profiles of a single exposure and/or from minor variations among multiple-epoch observations.

To investigate the dependence of the uncertainty on $v \sin i$, we used the difference between the individual $v \sin i$ values from the single-epoch observations and the associated mean value for the co-added spectra. There ought to be only minor differences among the rotation velocities of single-epoch exposures; their difference represents the uncertainty in our estimates. Royer et al. (2002a) found that the error in $\ln (v \sin i)$ can be approximated by a normal distribution, based on multi-epoch (48), high-SNR ( $>250$ ) observations of Sirius. This is because fast rotation blends the absorption lines and affects the accuracy of radial and rotation velocity measurements. Therefore, the variation was calculated in logarithmic space (see Figure 1). For $\ln (v \sin i)<4\left(v \sin i<50 \mathrm{~km} \mathrm{~s}^{-1}\right)$, the standard deviation (orange squares) exhibits a dependence on the $v \sin i$ value. This is at odds with the result of Royer et al. (2002b, 2007), who found 


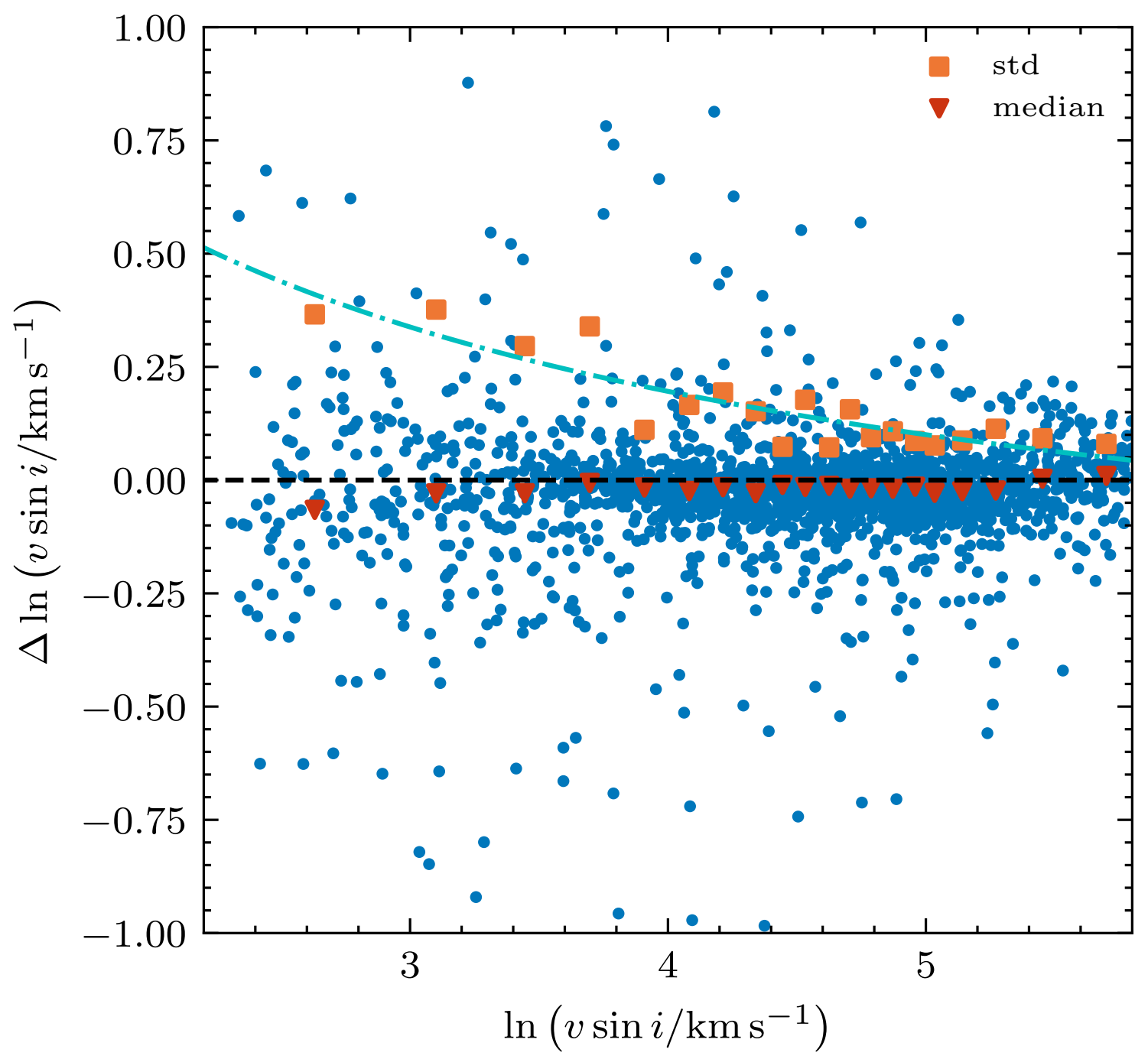

Figure 1. Variations of $v \sin i$ estimates from single-epoch observations with respect to the mean value. The data was analyzed using constant numbers in each bin. Orange squares represent the $1 \sigma$ uncertainties, $\Delta \ln v \sin i$, and red triangles represent the median value (bias). An inverse proportional function (cyan dot-dashed line) is overplotted to fit the standard deviations.

a uniform error value on a logarithmic scale. This is due to the differences between the methods used to estimate rotation velocities.

Because those earlier authors adopted the first zero of the Fourier transform of the line profiles (Carroll 1933; Gray 1976) based on high-resolution spectra $(R>20,000)$ to determine $v \sin i$, their estimates are less affected by resolution limitations. However, our data from the LAMOST MRS are unavoidably further broadened due to the finite resolution power of the instrument, which is known as instrumental broadening. The observed line profiles are a convolution of the true profiles and the instrumental profile, which makes it hard to estimate very slow rotation velocities and, hence, results in larger uncertainties for slow compared with fast rotators.

Based on our estimates using a spectrograph with $R \sim 4000$ (Sun et al. 2019), the detection limit for the MRS data $(R \sim 7500)$ is around $30 \mathrm{~km} \mathrm{~s}^{-1}$. For these slow rotators, the relative uncertainties are comparable to the absolute values, and so such data should be used with caution. We used an inverse proportional function to fit the standard deviations. The median values of $\Delta \ln v \sin i$ are also presented as red triangles in Figure 1. For stars with rotation velocities above the detection limit, the relative bias- $\operatorname{Bias}(v \sin i) / v \sin i$-is smaller than $3 \%$.

\section{CONTAMINATION}


The rotation behavior of our sample stars might not be solely the result of their own evolution but it could also be affected by several external processes. Therefore, possible polluters need to be discarded first. In this section, we present two possible contaminants: binary and CP stars. We check for the incidence of periodic variables and star cluster members in Section 3.3 and Section 3.4, respectively. We then discuss the different rotation properties of these contaminants and the effects of possibly having missed contaminants on the rotation distribution of 'normal' (single, non-CP, non-variable, non-cluster member) stars. Following removal of all identifiable contaminants, we are left with 33,655 normal stars (see Section 4.2).

Binaries, particularly close binaries, can drastically affect the rotation properties of both of their components through tides, mass transfer, or even mergers (de Mink et al. 2013). Tidal interactions tend to synchronize stellar and orbital rotation, transferring the stellar spin angular momentum to the orbital angular momentum of the binary system. Mass transfer from the donor star can spin up the accreting star and cause it to approach the break-up limit. Mergers may also change stellar rotation, although this is the least well-understood process.

CP stars represent another possible contaminant type in our sample. They are early-type MS stars exhibiting anomalous chemical abundances. According to Preston (1974)'s classification, they can be divided into four subgroups: metallic line (Am), magnetically peculiar (mAp), stars with enhanced Hg II and Mn II (HgMn), and He-weak stars. Most CP stars exhibit slow rotation, but the distribution may differ among subgroups. Abt \& Morrell (1995) found that virtually all Am and mAp stars have equatorial rotation velocities below $120 \mathrm{~km} \mathrm{~s}^{-1}$.

\subsection{Contamination by binary stars}

If a binary system's stellar rotation is modified by interactions between the components, that should also affect the system's radial velocity, $v_{R}$. Variations in the latter could be identified through Doppler shifts in the case of spectroscopic binaries. Ideally, such shifts should be periodic, following the orbital period, which could be confirmed by means of multi-epoch, high-cadence observations. However, only $1 \%$ of our sample objects have been observed more than 10 times, while most $(>60 \%)$ only have three epochs of observations. Therefore, we used the variations in $v_{R}$ between single-exposure spectra to tentatively infer binarity.

Relative variations were estimated following Xiong et al. (2021, in prep.). We relied on relative variations to avoid systematic errors in the absolute $v_{R}$ measures (Huang et al. 2018). In brief, we adopted the single-exposure spectrum with the highest SNR as our spectral template and calculated $\Delta v_{R}$ for the other spectra through cross-correlation. To differentiate binaries from single stars, we introduced $\sigma_{\text {detect }}$ (Sana et al. 2013), i.e. the ratio of the relative $\Delta v_{R}$ to its uncertainty. We considered a star to be a binary system if any pair of single-exposure spectra met the following condition:

$$
\sigma_{\text {detect }}=\frac{\left|v_{i}-v_{j}\right|}{\sqrt{\sigma_{i}^{2}+\sigma_{j}^{2}}}>C_{1} \text { and }\left|v_{i}-v_{j}\right|>C_{2},
$$

where $\left|v_{i}-v_{j}\right|$ is the maximum difference between these measurements. The latter criterion was introduced to exclude fake binary stars, i.e. stars with intrinsic variability that may be caused by photospheric or wind effects.

We adopted $\left(C_{1}=4, C_{2}=16\right)$ as our selection criteria for binary systems. We detected 4955 binaries from a sample of 36,620 candidates $\left(N_{\text {obs }} \geqslant 3\right)$. The corresponding binary fraction for this sample is $(13.5 \pm 0.3) \%$. This is lower than the binary fraction observed for O-type (Sana et al. 2013) and early-B type stars (Dunstall et al. 2015; Sana 2017), but consistent with Guo et al. (2021)'s result for late-B and early-A type stars. Note that a large fraction of binaries will remain undetected. Guo et al. (2021) used a Monto Carlo method to correct for observational biases and estimated that the intrinsic binary fraction for our sample could be as high as about $40 \%$. This type of analysis is not necessary for the context in which we are looking for contaminants. Our method to detect spectroscopic binaries is most sensitive to close binaries that could influence the stellar rotation rates of their components. Any missing binaries are mostly long-period systems, which are not expected to affect the rotation rates of their individual components.

\subsection{Contamination by CP Stars}

Identification of CP stars relies on strong (or weak) absorption lines of certain elements in their spectra. However, this is currently not feasible for our sample. First, the wavelength coverage of the LAMOST MRS is rather narrow and misses important lines that are used to identify anomalies (e.g., the Ca II H and K lines). However, a detailed analysis of the chemical abundances of early-type stars based on our MRS spectra is beyond the scope of this study, and we defer the identification of CP stars using the same data to future papers. 
Instead, we used the pre-compiled catalogs of Qin et al. (2019), Hümmerich et al. (2020), and Paunzen et al. (2021) to identify Am, mAp, and $\mathrm{HgMn}$ stars (the most frequent CP subgroups) in our sample. These three papers are all based on previous LAMOST DRs, which hence enables us to better estimate the frequency of CP-star occurrence. Qin et al. (2019) used a random forest machine-learning algorithm trained on the sample from the study of Hou et al. (2015) to search for Am stars from LAMOST DR5 high-SNR (> 50) early-type LRS spectra and they also used the equivalent width of the $4077 \AA$ line, a blended line of Sr II, Cr II, and Si II, to label mAp candidates. We reselected our sample following their selection criteria, and cross-matched our sample with their catalog. We found 405 Am stars and $24 \mathrm{mAp}$ candidates in a subsample of 8051 objects. Hümmerich et al. (2020) and Paunzen et al. (2021) applied a different approach based on MKCLASS to their DR4-based sample. We obtained $46 \mathrm{mAp}$ and 12 HgMn stars among 7676 candidates by cross-matching the DR4 data with their catalogs. Note that the selection of the subsamples in the latter cases is not the same as that described by Hümmerich et al. (2020) and Paunzen et al. (2021), who used Gaia DR2 colors, as well as spectral types, to perform early-type sample selection. We also cross-matched our sample with Renson \& Manfroid (2009), a catalog compiled from the literature. Ultimately, we identified $522 \mathrm{Am}, 115 \mathrm{mAp}, 13 \mathrm{HgMn}$, and 1 He-weak stars.

The apparent detection rate of Am stars in our subsample is around $5 \%$, which is similar to that reported by Qin et al. (2019). However, given that Am stars are mostly found around $T_{\text {eff }} \sim 7750 \mathrm{~K}$, the detection rate based on a sample with a wide range of temparture does not properly reflect the incidence. In the most likely temperature range, the frequency of occurrence is more than $15 \%$, but it is still lower than the values reported in previous studies (Preston 1974; Abt 1981; Zorec \& Royer 2012). This is probably due to Qin et al. (2019)'s strict screening, since they conducted additional manual identification based on spectral features associated with chemical peculiarity.

\subsection{Contamination by periodic variables}

Our comparison catalog of periodic variables was recently published by Chen et al. (2020). The authors searched for and classified 781,602 variables into 11 main types based on the Zwicky Transient Facility (ZTF; Bellm et al. 2019) DR2, which covers the northern sky. We also cross-matched our sample with a combination of other catalogs, including the Wide-field Infrared Survey Explorer (WISE, Chen et al. 2018), the Asteroid Terrestrial-impact Last Alert System (ATLAS, Heinze et al. 2018), the All-Sky Automated Survey for Supernovae (ASAS-SN, Jayasinghe et al. 2018), and the Catalina survey (Drake et al. 2014). We found that our sample contained 325 periodic variables.

\subsection{Contamination by cluster members}

One of the LAMOST-II MRS survey's science goals pertains to open clusters. The survey's target sample includes 18 open cluster-related fields, containing several tens of open clusters (Liu et al. 2020). It has been reported that the distribution of the rotation velocities of cluster members might be different from that of field stars (Huang \& Gies 2006), in the sense that cluster B-type stars contain fewer slow rotators relative to field B-type stars. Although Huang et al. (2010) argued that the lower rotation rates of the field stars are primarily the result of evolutionary spin-down changes, we removed any cluster members to retain a clean sample of early-type field stars. To do so, we adopted the cluster member catalog of Cantat-Gaudin \& Anders (2020), who applied a membership assignment procedure to the Gaia DR2 dataset and identified members of 1481 clusters. We found that our sample included 726 cluster members, equivalent to less than $2 \%$ of the total sample.

\section{5. $v \sin i$ contamination}

Even though we have tried to filter out most contaminants, a fraction may have been missed by our decontamination method, while the (poor) decontamination completeness may compromise estimates of the rotation distribution. It is, therefore, necessary to investigate the rotation properties of the contaminants discussed in the previous sections.

In Figure 2, we present $v \sin i$ histograms for the normal stars as well as the contaminants. Only CP stars (green) exhibit a significant deviation, in the sense that they are mostly slow rotators. The other objects display similar profiles as the normal stars (note that cluster members may exhibit a slightly different $v \sin i$ distribution). As long as the $v \sin i$ contamination is similarly distributed as the $v \sin i$ distribution of the normal stars, the overall rotation distribution will not be affected even if a fraction of contaminants remains unidentified in our final sample. As for the cluster subpopulation, we note that the completeness of this catalog should be close to unity since it was derived using high-precision proper motion data from Gaia.

CP stars tend to be slow rotators (e.g., Preston 1974; Abt \& Morrell 1995). Chemical peculiarities in Am and mAp stars are presumed to have been caused by gravitational settling and radiative levitation, which can occur in 


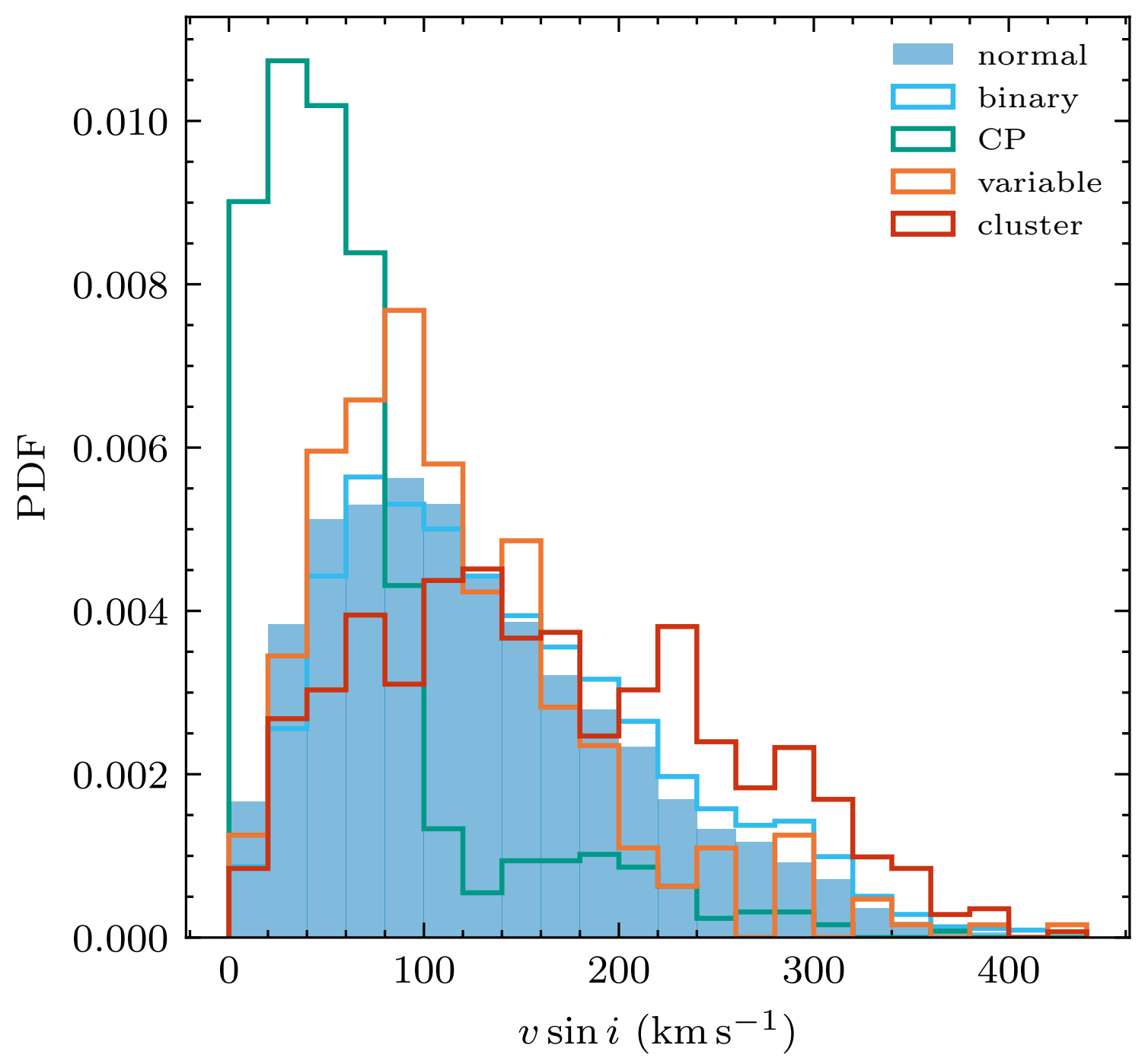

Figure 2. $v \sin i$ distributions of (blue) normal stars, (cyan) binary stars, (green) CP stars, (orange) periodic variables, and (red) cluster members.

stable atmospheres associated with slow rotation rates. However, the existence of slow rotators but without chemical anomalies challenges the connection between their chemical peculiarities and stellar rotation. We confirm their special rotation properties in Figure 3, where it is clear that most of CP stars have small $v \sin i$ values, which is rather different from the behavior of the normal stars in the $M$ versus $v \sin i$ diagram. Since most of the CP stars detected are Am and $\mathrm{mAp}$ stars, only these two subtypes are shown. Am stars (blue) span a narrow mass range from $1.5 \mathrm{M}_{\odot}$ to $2.0 \mathrm{M}_{\odot}$; they are less massive than mAp stars. This is consistent with the classification scheme of CP subtypes. In the right panel, we present the normalized probability density function (PDF) of the $v \sin i$ values of Am and $\mathrm{mAp}$ stars. We note that 10-20\% of these CP stars have relatively large rotation velocities $\left(v \sin i>120 \mathrm{~km} \mathrm{~s}^{-1}\right)$, which was also noted by Netopil et al. (2017). The presence of such rapidly rotating CP stars, if confirmed, would shed new light on the possible role of stellar rotation in the context of CP star formation (Abt 2009).

The top panel of Figure 3 shows the number fraction of CP stars $\left(N_{\mathrm{CP}} / N_{\text {total }}\right)$ in each mass bin from $1.6 \mathrm{M}_{\odot}$ to $4.0 \mathrm{M}_{\odot}$ with a bin width of $0.2 \mathrm{M}_{\odot}$. The average CP fraction is around $1-2 \%$ across the mass range, and any differences in CP fraction may be attributed to the various subtypes of CP stars populating the different mass domains. However, based on estimates of the frequency of CP stars by Preston (1974), Abt (1981), and Zorec \& Royer (2012), 15-30\% of solar neighborhood stars ( covering $7000 \mathrm{~K}<T_{\text {eff }}<7800 \mathrm{~K}$ ) are CP stars. It thus appears that a fraction of missing CP stars may remain undetected in our sample, and they may bias the rotation distribution of the normal stars. However, 

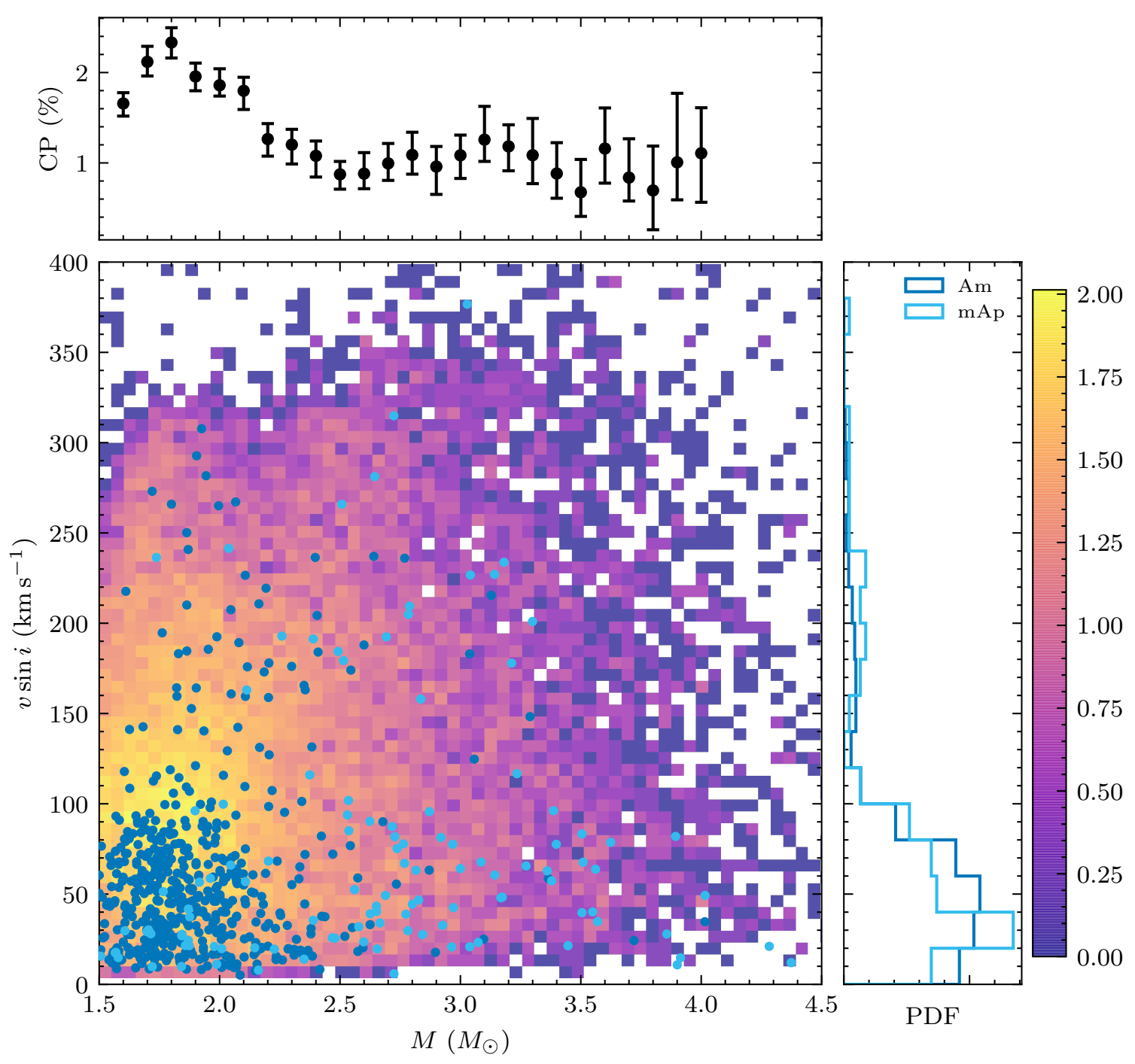

Figure 3. $v \sin i$ as a function of stellar mass, $M$, for CP stars. The corresponding distribution of normal stars is presented in the background. Am and mAp stars are represented by blue and cyan circles, respectively. The right panel shows histograms of the $v \sin i$ distribution of these two subpopulations. The top panel shows the number fraction of CP stars in each mass bin, from $1.6 \mathrm{M}_{\odot}$ to $4.0 \mathrm{M}_{\odot}$ with a bin width of $0.2 \mathrm{M}_{\odot}$.

statistical corrections are difficult and imprecise, because the observed incidence may depend on the temperature as well as the detection method.

\section{DISTRIBUTIONS OF EQUATORIAL ROTATION VELOCITIES}

To obtain the equatorial rotation velocities, $v$, we need to correct the projected rotation velocities $v \sin i$ for the effects of inclination, $i$. For individual stars, this is only feasible in rare cases, e.g. for eclipsing binaries (where strong tidal effects ensure that the rotation axis is parallel to the orbital axis) and for stars with a rotational modulation signal in their light curves, among others. However, we can correct for projection effects statistically under the assumption of randomly oriented rotation axes (Abt \& Morrell 1995; Royer et al. 2007; Zorec \& Royer 2012).

To estimate the distribution of the equatorial rotation velocities, we followed Royer et al. (2007) and Zorec \& Royer (2012), whose method can be summarized in three steps:

1. We first smooth the distribution using a Gaussian kernel. The selection of the bandwidth of the kernel-smoothing window is done using Silverman's rule of thumb (Silverman 1986) which minimises the mean integrated squared 
error

$$
h=\left(\frac{4 \hat{\sigma}}{3 n}\right)^{\frac{1}{5}}
$$

where $h$ is the bandwidth of the smoothing parameter, $\hat{\sigma}$ is the standard deviation of the samples, and $n$ is the sample size.

2. We then recover the PDF of the true projected rotation velocities, $\theta \equiv \widehat{v \sin i}$, through rectification of the error law. As our measurements are subject to random uncertainties, the observed $v \sin i$ represents the maximum of the probability distribution, whereas $\sigma_{v \sin i}$ is the width of the distribution. Royer et al. (2007) assumed that the error in $\ln (v \sin i)$ was normally distributed, with a mean of 0 and a variance of $(\ln (1+\alpha))^{2}$, where the coefficient $\alpha=0.1$ is a constant. However, as shown in Figure 1, our estimates of $v \sin i$ are susceptible to instrumental broadening for small $v \sin i$. Therefore, $\alpha$ depends on the value of $v \sin i$, which must hence be implemented into our calculation. The deconvolution is carried out using the Lucy (1974) iterative technique.

Although our $v \sin i$ estimates are consistent with literature values up to $\sim 400 \mathrm{~km} \mathrm{~s}^{-1}$, they could be affected by temperature and gravity inhomogeneities introduced by rapid rotation. Gravity darkening suppresses the equatorial region's contribution to the rotational broadening, which has been discussed extensively in the context of Be stars, i.e. B-type stars exhibiting Balmer-line emission (e.g. Townsend et al. 2004). Nonetheless, a correction for this underestimation is only necessary if the rapid rotators represent a statistically significant fraction (Zorec \& Royer 2012).

3. Following correction for the error law, the final step is to correct the distribution for inclination effects. Under the assumption of a random distribution of rotation axes, the PDF of the corrected projected rotation velocities, $\Psi(\theta)$, becomes

$$
\Psi(\theta)=\int \gamma(v) P(\theta \mid v) \mathrm{d} v=\int \gamma(v) \frac{\theta}{v} \frac{H(v-\theta)}{\sqrt{v^{2}-\theta^{2}}} \mathrm{~d} v,
$$

where $\gamma(v)$ is the true equatorial rotation velocity distribution and $P(\theta \mid v)$ is the conditional probability. $H(x)$ represents the Heaviside step function, which is 1 if $x \geq 1$ and otherwise 0 .
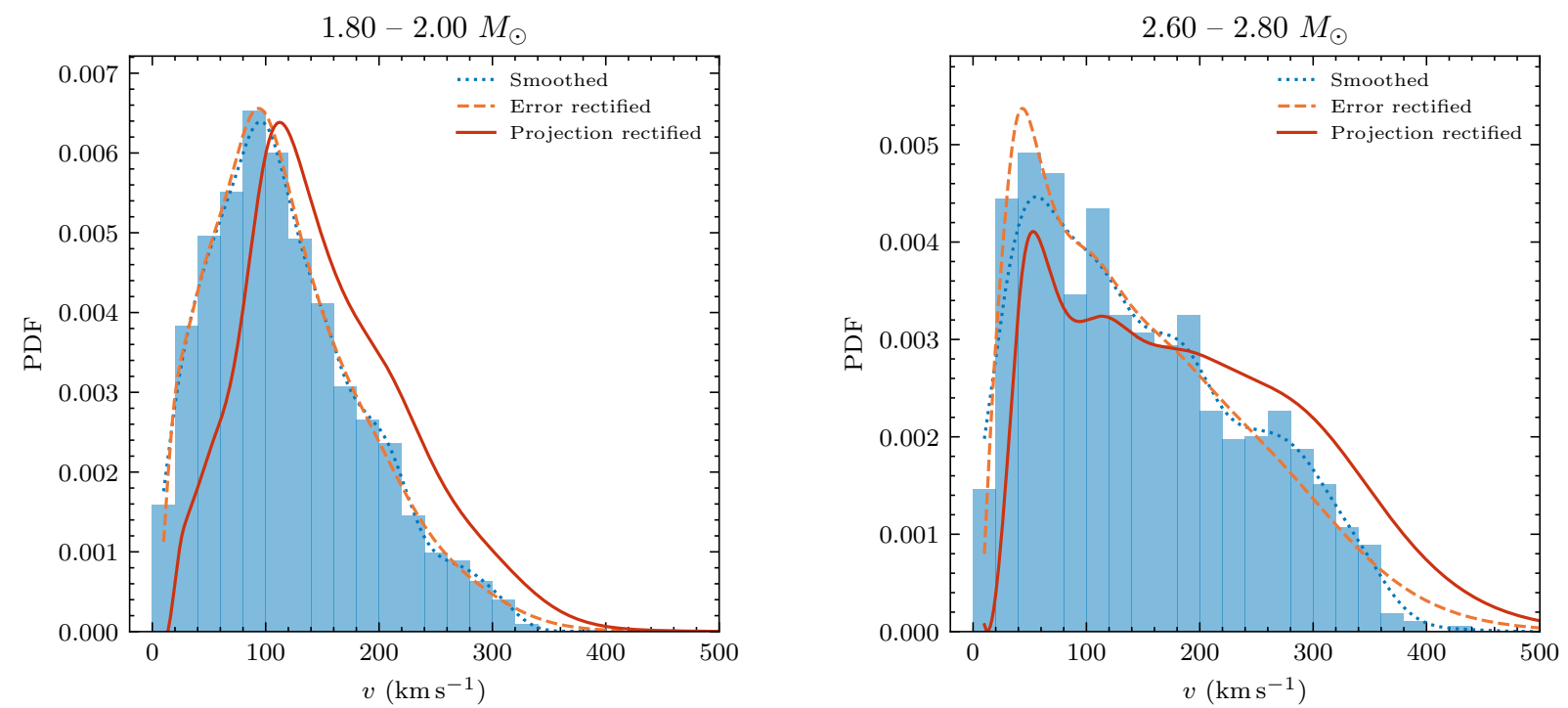

Figure 4. Distributions of rotation velocities of normal stars in different mass bins. (left) $1.8-2.0 \mathrm{M}_{\odot}$; (right) $2.6-2.8 \mathrm{M}_{\odot}$. The cyan histograms represent the observed $v \sin i$ distribution using a bin width of $20 \mathrm{~km} \mathrm{~s}^{-1}$, while the blue dotted lines are the corresponding smoothed distributions. The error-rectified distributions are shown as the orange dashed lines. The final products - projection-corrected equatorial velocity distributions - are shown as the red solid lines.

We provide two examples for different mass ranges (from 1.8 to $2.0 \mathrm{M}_{\odot}$ and from 2.6 to $2.8 \mathrm{M}_{\odot}$ ) to illustrate the process of deconvolution: see Figure 4. The original observed distribution of $v \sin i$ is shown as the cyan histograms 
with a bin width of $20 \mathrm{~km} \mathrm{~s}^{-1}$. Its smoothed $v \sin i$ distribution, error-corrected true projected rotation velocity $(\theta)$ distribution, and projection-rectified true equatorial rotation velocity $(v)$ distribution are represented by the blue dotted line, the orange dashed line, and the red solid line, respectively.

These two mass ranges were selected to represent the typical situation in the less massive $\left(M<2.5 \mathrm{M}_{\odot}\right)$ and more massive $\left(M>2.5 \mathrm{M}_{\odot}\right)$ regimes. Zorec \& Royer (2012) found that less massive stars have a unimodal equatorial velocity distribution, while more massive stars trace a bimodal distribution. Our results are largely consistent with previous results, except for several significant differences:

- The velocity distribution of the less massive stars (Figure 4, left) shows a peak velocity at $v \approx 110 \mathrm{~km} \mathrm{~s}^{-1}$, which is $\sim 40 \mathrm{~km} \mathrm{~s}^{-1}$ smaller than the peak velocity reported by Zorec \& Royer (2012) for the same mass range.

- For the more massive stars, we found an excess of stars with $v \sin i<100 \mathrm{~km} \mathrm{~s}^{-1}$, which results in a broad and rather flat rectified distribution. This is different from the bimodal distribution found by Abt \& Morrell (1995) and Zorec \& Royer (2012). The distribution in the right panel of Figure 4 could be interpreted as showing two populations, with one group representing slow rotators that share similar rotation velocities, and a second group comprising fast rotators whose velocities are widely spread. If this were correct, we could still claim a bimodal distribution for our more massive sample. Note that the peak velocity of the slow rotators is similar to that found by Zorec \& Royer (2012). However, the number fraction of slow rotators is rather distinctive in both papers. Zorec \& Royer (2012) found that the maximum PDF for slow rotators is smaller than that for fast rotators, and the number fraction for the former population is less than $20 \%$. However, slow rotators provide a more distinct contribution to our sample.

- Zorec \& Royer (2012) claimed that the sum of two Maxwellian functions can fit the rectified distributions. They used the derived Maxwellian functions to quantify the typical velocities and number fractions of slow and fast rotators. This was based on Deutsch (1967), who showed that the distribution of $v$ can be described by Maxwellian functions. However, we note that this practice may not always yield a reliable representation of the $v$ distribution, especially for the rapidly rotating population.

We suggest that the difference in peak velocity for the less massive stars and the excess of slow rotators among more massive stars can be attributed to missed contamination by CP stars. As discussed in Section 3.5, CP stars are mainly slow rotators and we expected to have $\sim 15 \%$ of such stars misclassified as normal stars. Given these missing CP stars, the peak velocity of the low-mass stars could be biased toward the lower end and a possibly bimodal distribution could be hidden. This may also explain an excess of slow rotators compared with previous studies.

However, recent papers have suggested the existence of a population of slowly rotating normal early-type stars (Sikora et al. 2020; Qin et al. 2021). Most of these studies are based on photometric variability caused by the inhomogeneous structure of the stellar surface, which could be used to infer the rotation period. Qin et al. (2021) searched for lowvelocity rotating normal (non-CP) A-type stars from the LAMOST-Kepler project and found a significant fraction of slow rotators among normal A-type stars. Such a contradiction is also reported for the 30 Doradus starburst region in the Large Magellanic Cloud (LMC). The O-type stellar sample (Ramírez-Agudelo et al. 2013) shows a similar rotation distribution as the right panel of Figure 4), exhibiting an excess of slow rotators, while the B-type stellar sample (Dufton et al. 2013) present a bimodal population of very slow and fast rotators, with few stars rotating at the low-velocity peak seen for the O-type stars. These controversies may suggest that previous studies of the rotation properties of early-type stars may have missed a significant number of slow rotators.

\section{DISTRIBUTIONS OF ROTATION RATES}

For a single star that is not affected by binary interactions, we can generalize the formalism of the observed rotation velocity, $v$, by writing out its dependence on mass and stellar age:

$$
v\left(M, \omega_{\text {init }}, t / t_{\mathrm{MS}}\right),
$$

where the stellar age is expressed as the relative age and the initial rotation rate is given by $\omega_{\text {init }}=\Omega / \Omega_{\text {crit }}($ Georgy et al. 2013). The parameter $\Omega$ is the angular velocity and $\Omega_{\text {crit }}$ is the critical velocity where the surface gravity can no longer maintain equilibrium with the centrifugal motion. Since $v$ changes as a function of time, and also depends on the stellar mass, the parameter $v$ itself is not suitable for comparison among samples with different properties. 


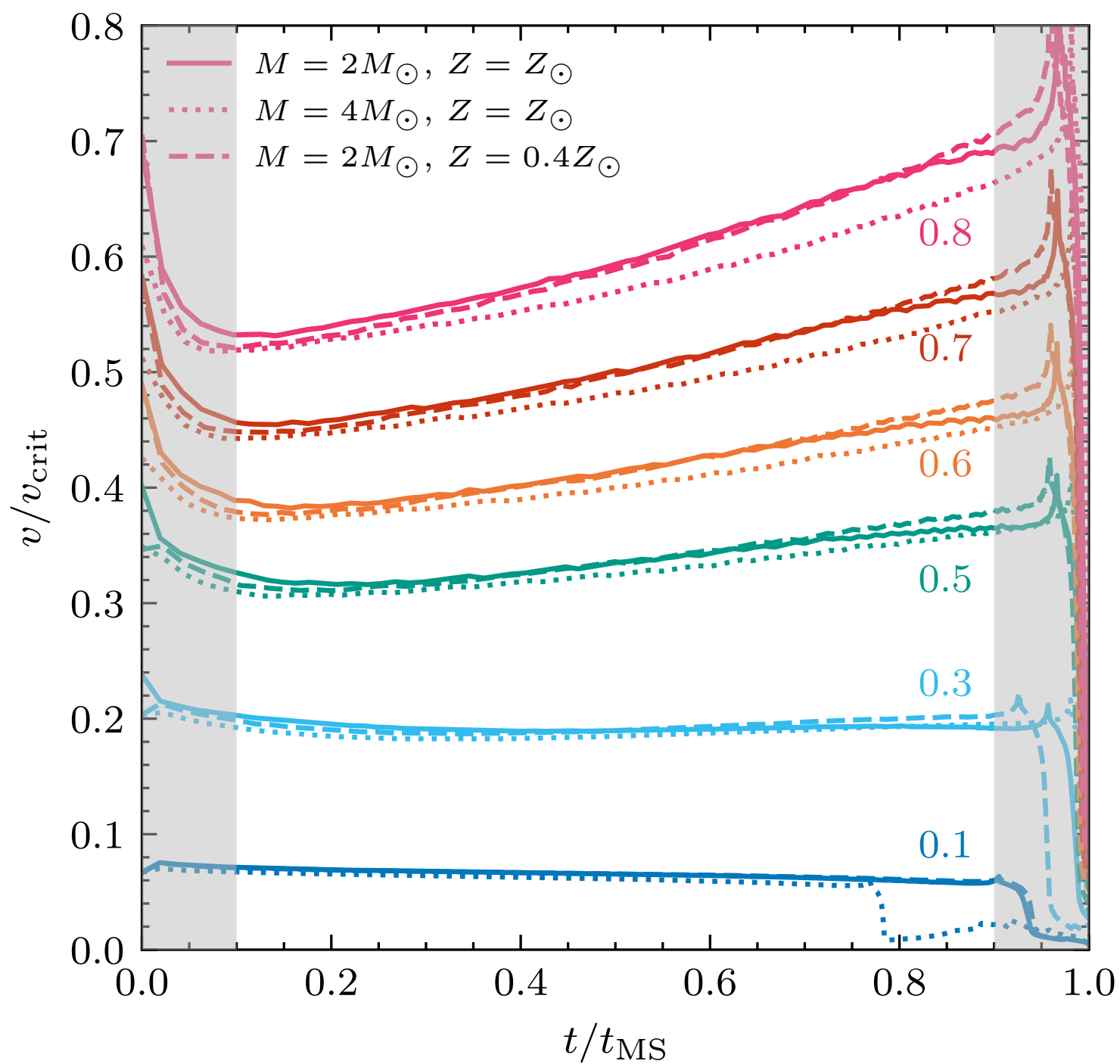

Figure 5. $v / v_{\text {crit }}$ evolution (Georgy et al. 2013) for different initial rotation rates $(0.1,0.3,0.5,0.6,0.7,0.8)$. Solid lines represent a $2 \mathrm{M}_{\odot}$ star of solar abundance, dotted lines represent a $4 \mathrm{M}_{\odot}$ star of solar abundance, while dashed lines represent a $2 \mathrm{M}_{\odot}$ star of $0.4 \mathrm{Z}_{\odot}$. The region where $v / v_{\text {crit }}$ shows a strong dependence on relative age is marked in gray. For $0.1 \leq t / t_{\mathrm{MS}} \leq 0.9$, $v / v_{\text {crit }}$ could be used as a proxy for the initial rotation rate.

We suggest this dependence on mass and age can be alleviated by introducing $v / v_{\text {crit }}$ (e.g. Netopil et al. 2017). For slow rotators, this ratio is almost constant, particularly for rigid rotators on the MS (Zorec \& Royer 2012). We illustrate this by exploring the evolutionary models of the Geneva group (Ekström et al. 2012; Georgy et al. 2013). In Figure 5, we show the $v / v_{\text {crit }}$ evolution for different initial rotation rates. The ratio is almost constant for $0.1 \leq t / t_{\mathrm{MS}} \leq 0.9$ if $\omega<0.5$. For fast rotators, the ratio exhibits a mild increase with time. This is to account for the enhanced 'mechanical' mass loss in the equatorial region as a star reaches the critical velocity. Even though the $v / v_{\text {crit }}$ ratio unavoidably depends on evolution, it is still a good proxy for the initial rotation rates and only weakly dependent on mass, age, or metallicity. The earliest phase, $t / t_{\mathrm{MS}} \leq 0.1$, corresponds to the time taken to reach a quasi-equilibrium state, while the late phase at $t / t_{\mathrm{MS}} \geq 0.9$ represents the star's deceleration as it evolves off the MS.

Therefore, we apply a similar analysis as in Section 4 to the $v / v_{\text {crit }}$ ratio. Considering the significant $v / v_{\text {crit }}$ variations that occur during the early and late phases of MS evolution, we limit our analysis to stars characterized by $0.1 \leq t / t_{\mathrm{MS}} \leq 0.9$, which covers $86 \%$ of our sample. The critical velocity was adopted from Georgy et al. (2013) by interpolating the grid of masses and relative ages for a given metallicity. Note that rapid rotation can increase the MS lifetime (Georgy et al. 2013) by transporting fresh hydrogen into the core. Depending on the initial rotation rate, the 
stellar lifetime could be extended by up to $30 \%$. This was not considered in estimating the ages for our sample based on the non-rotating stellar evolution models. Nevertheless, estimates of the relative age, which represents the fraction of a star's lifetime spent on the MS, is not affected by this effect.

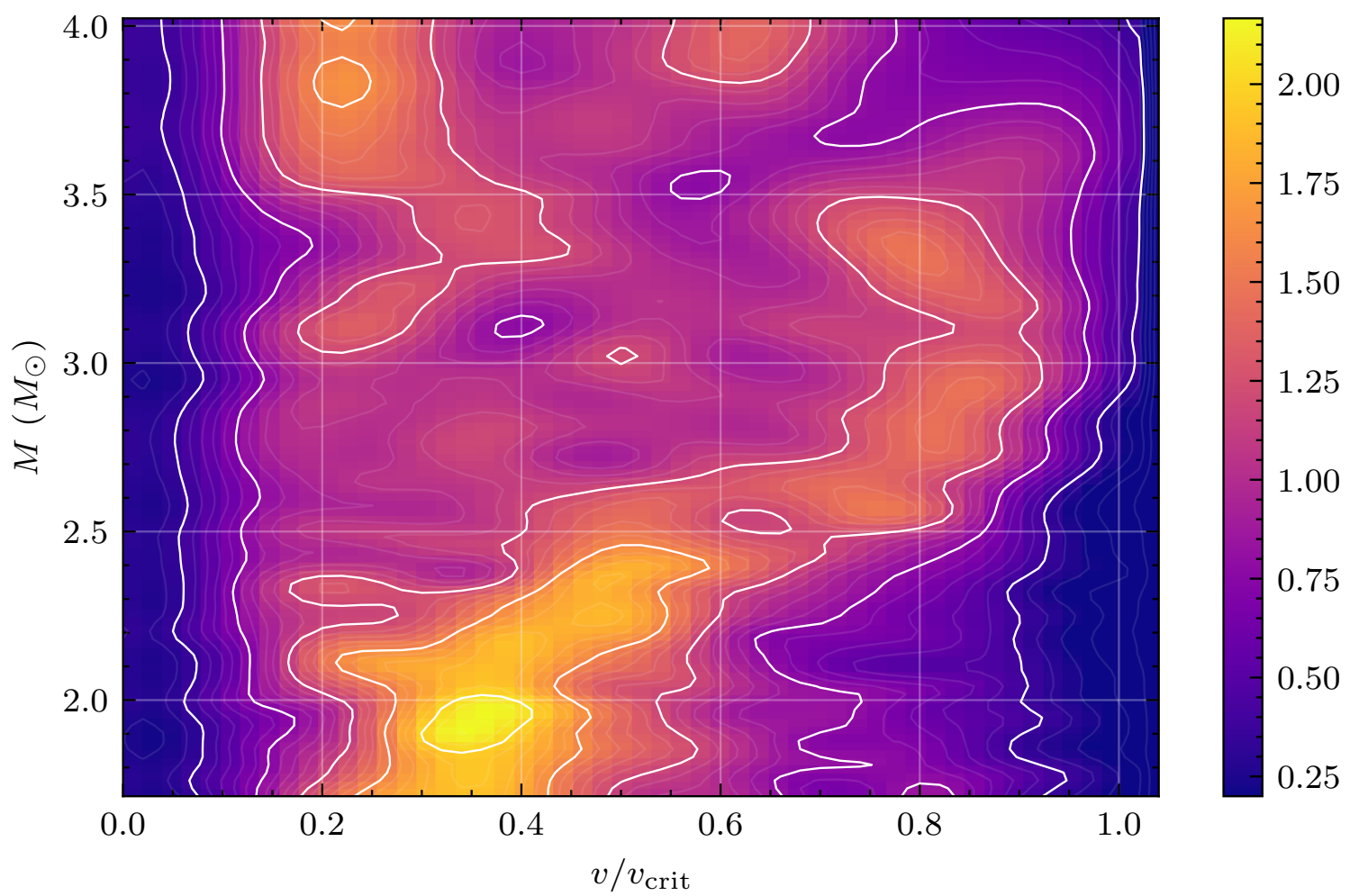

Figure 6. Distribution of $v / v_{\text {crit }}$ for normal stars as a function of stellar mass, color-coded by density in the one-dimensional normalized distribution. Iso-density contours are shown as white curves.

An overview of the distribution of $v / v_{\text {crit }}$ as a function of mass for normal stars is displayed in Figure 6 . Using the same color scale as before, the figure is color-coded by the relevant number density pertaining to the one-dimensional normalized distribution.

For less massive stars $\left(M<2.5 \mathrm{M}_{\odot}\right)$, the distribution of $v / v_{\text {crit }}$ is unimodal, with a significant concentration around the peak velocity, particularly in the mass range $1.8 \mathrm{M}_{\odot} \leq M \leq 2.0 \mathrm{M}_{\odot}$. The velocity ratio exhibits a clear dependence on stellar mass, in the sense that it increases from $\sim 0.3$ to $\sim 0.55$ as mass increases, which is consistent with Zorec \& Royer (2012). However, a lack of slow rotators $\left(v \leq 100 \mathrm{~km} \mathrm{~s}^{-1}\right)$ previously reported for this mass range is not seen in our data. Since Zorec \& Royer (2012) found that slow rotators are underrepresented even when they are not removed from the sample, they claimed that this lack is unlikely due to contamination by CP stars. Instead, they attributed it to sampling effects in the observed volume. However, in our sample we note that there are fewer slow rotators following our selection of a subsample with SNR $>50$ in the $g$ band and overlapping with LAMOST DR5 to reproduce the parent sample of Qin et al. (2019), Hümmerich et al. (2020), and Paunzen et al. (2021). This way, we can reduce the impact of contamination by CP stars by generating a cleaner sample than that used for Figure 6 . However, this relative absence of slow rotators only affects stars with $v / v_{\text {crit }}<0.2\left(v \leq 70 \mathrm{~km} \mathrm{~s}^{-1}\right)$; a significant fraction of stars with $70 \mathrm{~km} \mathrm{~s}^{-1} \leq v \leq 100 \mathrm{~km} \mathrm{~s}^{-1}$ remains. Therefore, we conclude that, even if the reported relative lack of stars is real, it must be affected by contamination by CP stars but does not extend to $v \sim 100 \mathrm{~km} \mathrm{~s}^{-1}$.

This trend between mass and $v / v_{\text {crit }}$ continues to develop as stars become more massive than $2.5 \mathrm{M}_{\odot}$ and may continue up to $\sim 3.0 \mathrm{M}_{\odot}$, where a mild turnover/upper limit is seen. The branch of slow rotators becomes prominent for $M>3.0 \mathrm{M}_{\odot}$, but the bimodality is already present for $M>2.5 \mathrm{M}_{\odot}$. The reason why the slowly rotating branch is not prominent in this figure is that it covers an extended distribution across $v / v_{\text {crit }}$. In fact, Zorec \& Royer (2012) found that a large fraction of stars have intermediate rotation velocities compared with the slow or fast rotators, and it is not until $M>3.0 \mathrm{M}_{\odot}$ that a gap between the slowly and rapidly rotating branches becomes apparent. The 


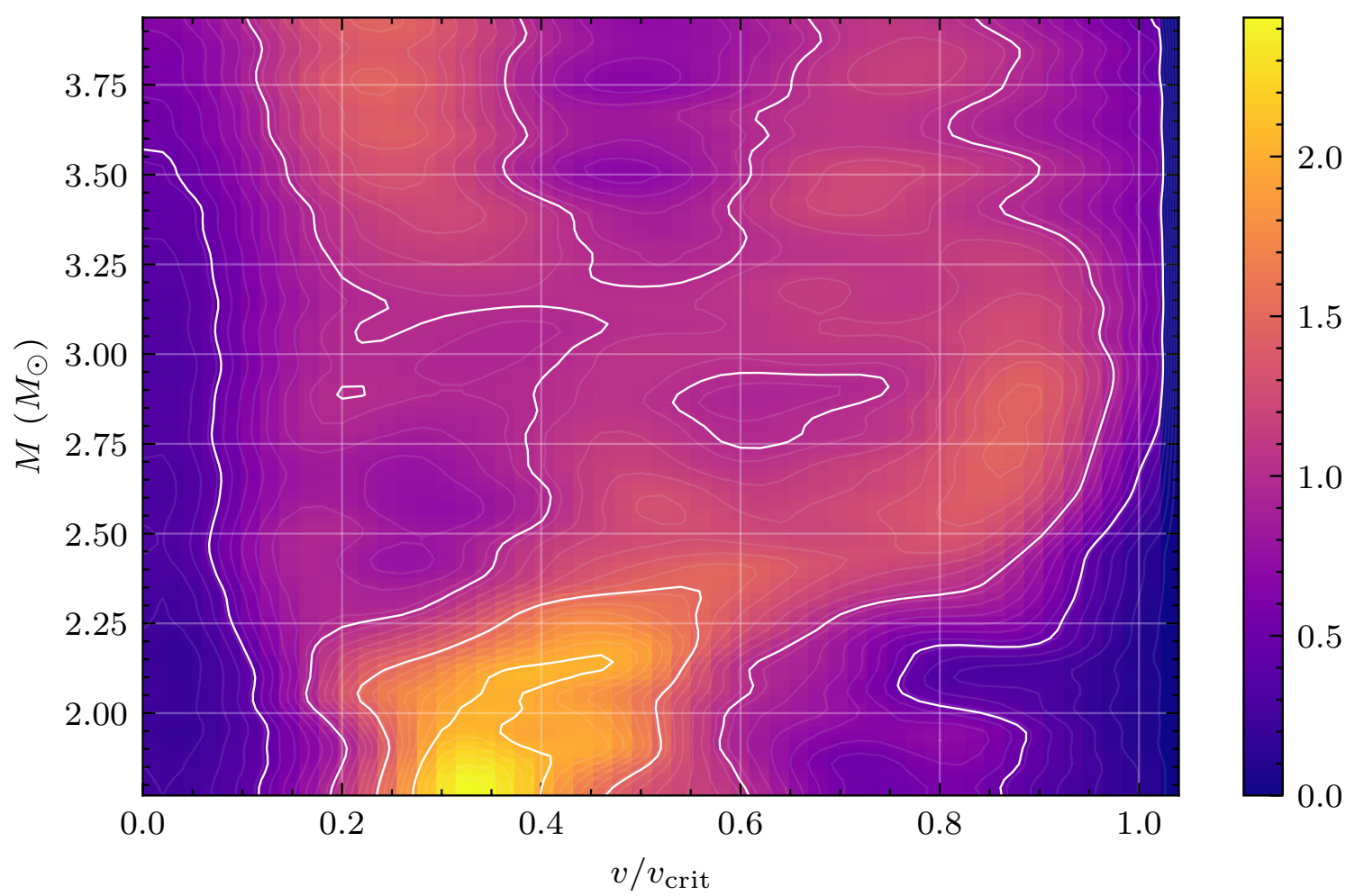

Figure 7. As Figure 6, but for a subsample with SNR $>50$ in the $g$ band, overlapping with LAMOST DR5. This subsample reproduces the parent sample of Qin et al. (2019), Hümmerich et al. (2020), and Paunzen et al. (2021) and reduces the impact of contamination by CP stars by generating a cleaner sample than that used for Fig. 6 .

connecting structure is better visible in Figure 7, where the slowly rotating branch has bifurcated from the rapidly rotating branch at $M \sim 2.5 \mathrm{M}_{\odot}$. For masses greater than $\sim 3.25 \mathrm{M}_{\odot}$, the gap between the slowly and rapidly rotating branches is well-established.

As stellar masses increase to beyond $\sim 3.6 \mathrm{M}_{\odot}$, our analysis suffers from poor sampling in the $\log T \operatorname{vs} \log L$ parameter space (Paper I, Figure 5). Massive MS stars in early evolutionary phases are missing in our sample. Nevertheless, we can make an educated guess of their properties based on the available information. If we assume that younger stars behave similarly as their older counterparts, it appears that more stars reside on the slowly rotating branch compared with stars with masses in the range $2.5 \mathrm{M}_{\odot} \leq M \leq 3.25 \mathrm{M}_{\odot}$.

A similar bimodal distribution in rotation period has been observed for late-type field dwarfs (e.g. McQuillan et al. 2013), mainly by the Kepler and K2 missions (Borucki et al. 2010). Gordon et al. (2021) detected a gap in the rotation periods for $0.57 \mathrm{M}_{\odot}<M<0.76 \mathrm{M}_{\odot}$ based on a sample of $\sim 70,000 \mathrm{~K} 2$ targets and attributed it to a departure from the Skumanich (1972) spin-down law. As a young star with its envelope initially decoupled from its core loses angular momentum due to magnetic braking, it could slow or even halt its spin-down if the core and envelope begin to recouple at a later time. Despite the differences between our and their samples in terms of the mass regime covered, such a mechanism might also occur in early-type stars (e.g. Zorec \& Royer 2012).

\section{DEPENDENCE ON METALLICITY}

Other than on mass, stellar rotation also depends on metallicity (e.g., Ekström et al. 2008). The meridional circulation cells that carry angular momentum from the inner regions to the surface become less efficient at lower metallicity. In addition, stellar winds also cause angular momentum losses. Wind strengths are greater for higher metallicities. The models of Georgy et al. (2013) and Szécsi et al. (2015) suggest that normally evolving stars with low mass loss (e.g., low metallicities) may increase their rotation during MS evolution and that the rotation distribution of early-type MS stars in lower-metallicity environments might be different from that in higher-metallicity environments. However, thus far, such differences have not been observed conclusively. Studies of rotational velocities of O- and B-type stars in the LMC and the Small Magellanic Cloud (SMC) (e.g., Penny et al. 2004; Penny \& Gies 2009; Dufton et al. 2019) 
found little compelling evidence for differences with metallicity. Hunter et al. (2008) found that massive stars (with masses greater than $8 \mathrm{M}_{\odot}$ ) in the $\mathrm{SMC}$ rotate faster than those in the solar neighborhood. (There is no significant difference between the distribution of rotation rates in the Galaxy and the LMC.) However, the analysis was hindered by field star contamination (similar to the situation in Section 3.4) because of the complicated composition of their sample, which includes target stars projected toward young clusters and a fraction of field stars.

To address the possible metallicity dependence, we selected metal-poor $([\mathrm{M} / \mathrm{H}]<-0.2 \mathrm{dex})$ and metal-rich $([\mathrm{M} / \mathrm{H}]>$ 0.2 dex) subsamples. Each comprises more than 5000 normal stars to ensure sufficient sample sizes for statistical analysis. Using the same procedures as discussed in Section 5, we analyzed their rotation distributions as a function of stellar mass.

The top and bottom panels of Figure 8 show the rotational profiles for metal-poor and metal-rich stars, respectively. There is a significant difference between both distributions, in the sense that metal-poor stars only exhibit a single branch of slow rotators, with $v / v_{\text {crit }}$ centered at 0.3-0.4, while metal-rich stars clearly show two branches of the stellar velocity ratio. The rapidly rotating branch is similar to the feature we saw in Figures 6 and 7 , but only for $M \geq 2 \mathrm{M}_{\odot}$. The velocity ratio of the less massive, metal-rich stars peaks at $v / v_{\text {crit }} \sim 0.2$. This is much smaller than previously observed for our entire sample. Moreover, the slowly rotating branch is also located at lower velocity ratios than that of the metal-poor subsample.

The shape and location of the velocity distribution pertaining to the metal-rich subsample could be affected by slowly rotating CP stars. Since most CP stars are metal-rich (e.g. Qin et al. 2019), there is a larger fraction of missing $\mathrm{CP}$ stars in this metal-rich subsample compared with both the entire sample and the metal-poor subsample, where contamination is expected to be minimal. Netopil et al. (2017) found a linear correlation between the masses and rotation rates of $\mathrm{mAp}$ stars, with $v / v_{\text {crit }}$ increasing from 0.08 to 0.17 as mass increases from $\sim 1.8 \mathrm{M}_{\odot}$ to $\sim 4.5 \mathrm{M}_{\odot}$ ( $\sim 30 \%$ scatter). This could be the reason for the low rotation rate of the slowly rotating branch as seen for the metal-rich subsample. Note that the incidence of $\mathrm{CP}$ stars is not large enough to completely explain the rotation characteristics for $M \leq 2 \mathrm{M}_{\odot}$. Thus, among less massive stars (which are most numerous), the rotation rates of normal metal-rich stars are lower than those for the metal-poor stars. The contribution of the rapidly rotating branch becomes obvious for $M \geq 2.5 \mathrm{M}_{\odot}$.

The lack of a rapidly rotating branch for the metal-poor subsample is intriguing. One possible explanation is that this feature is determined within the first few Myr of a star's lifetime through 'disk locking' (e.g. Bouvier et al. 1993). Magnetic interactions between a star and its accretion disk can remove angular momentum and form intrinsically slow rotators (Lin et al. 2011), and the longer disk lifetime results in slow rotation velocities on the MS (Bastian et al. 2020). However, Yasui et al. $(2009,2010)$ found that the near-infrared disk fraction of low-metallicity stars declines rapidly on timescales $<1 \mathrm{Myr}$, much faster than the $\sim 6 \mathrm{Myr}$ observed for solar abundance. If this were to also apply to our metal-poor subsample, and the initial rotation rates are determined by this mechanism, the metal-poor subsample should rotate faster than their metal-rich counterparts. This may explain the difference in velocity ratio in the slowly rotating branch between the metal-poor and metal-rich subsamples, but not the absence of a rapidly rotating branch.

Another possible mechanism to slow down stellar rotation at birth is magnetic braking (Meynet et al. 2011). The coupling of wind material and magnetic field lines that extend well beyond the stellar surface exerts a torque on the stellar surface layers and thus slows down rotation. Unlike magnetic fields in late-type stars, which are thought to be generated through dynamo action, magnetic fields in early-type stars are likely of fossil origin (Donati \& Landstreet 2009). Sanyal et al. (2017) proposed that high-mass $\left(M \geq 25 \mathrm{M}_{\odot}\right)$ stars develop envelope inflation during their lifetime on the MS, which is favorably correlated with metallicity. Therefore, stronger fossil fields are expected for massive stars with lower metallicity than for higher-metallicity stars under the fundamental physics law of flux conservation, where the surface magnetic field strength is expected to decrease as the stellar radius expands. If confirmed, magnetic braking could be more prominent in the metal-poor subsample. However, the incidence rate of magnetic fields in early-type MS stars is around 10\% within the solar neighborhood (Sikora et al. 2019), and thus the lack of a rapidly rotating branch cannot be explained exclusively by magnetic braking.

Instead, the lack of a rapidly rotating branch could also be related to the fact that the metal-poor subsample is generally much older than the full sample (see Figure 9). If the overall spin down of metal-poor stars is stronger than predicted by stellar evolutionary models and more (specific) angular momentum is lost during the MS phase, the rapidly rotating branch of the older metal-poor subsample may have been moderated and may even have merged with the slowly rotating branch. This would make it appear as a single population with slightly higher rotation rates than characteristic of the slowly rotating branch. 

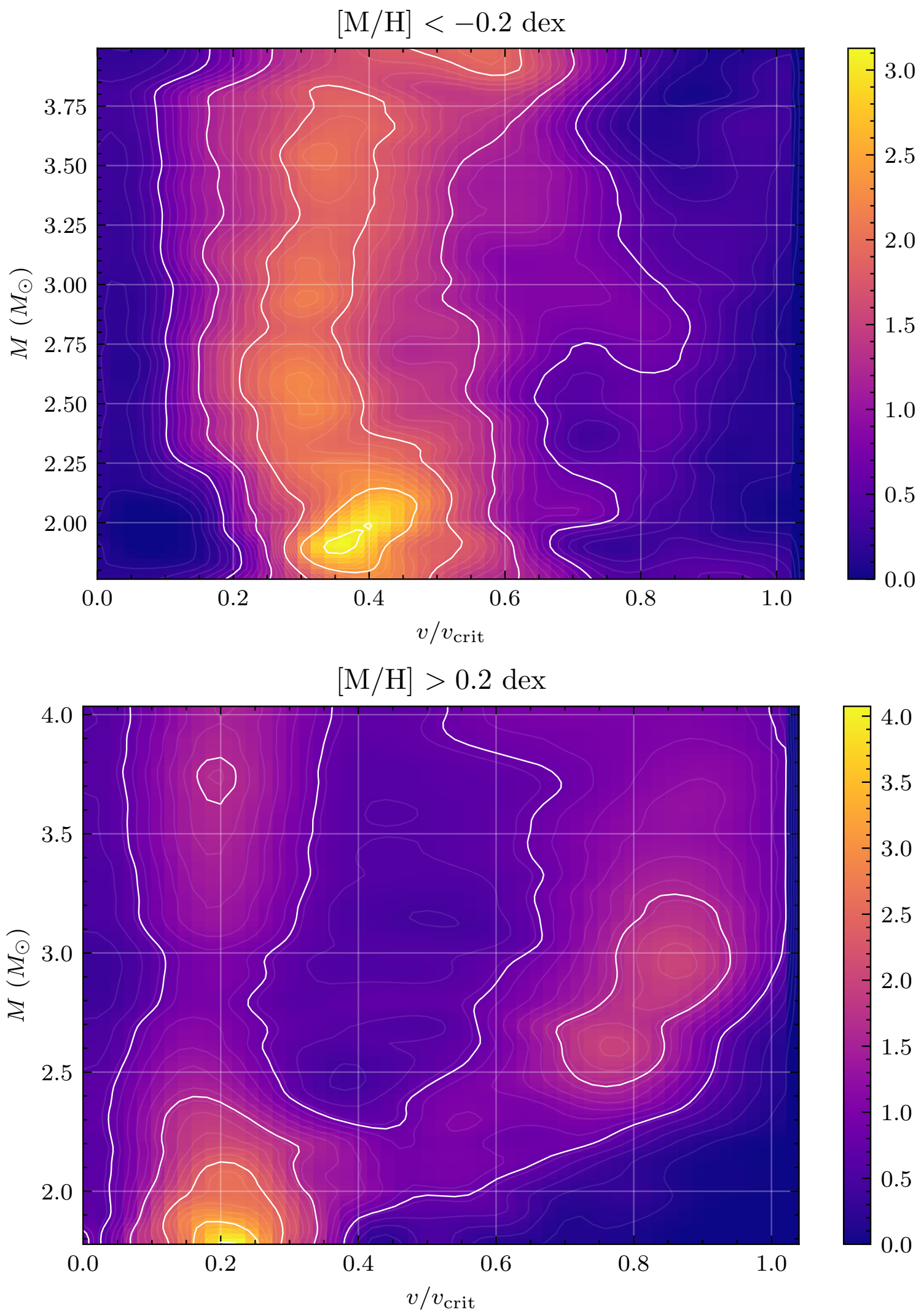

Figure 8. As Figure 6, but for different metallicity ranges. (top) Metal-poor $([\mathrm{M} / \mathrm{H}]<-0.2$ dex); (bottom) Metal-rich $([\mathrm{M} / \mathrm{H}]>0.2 \mathrm{dex})$. 


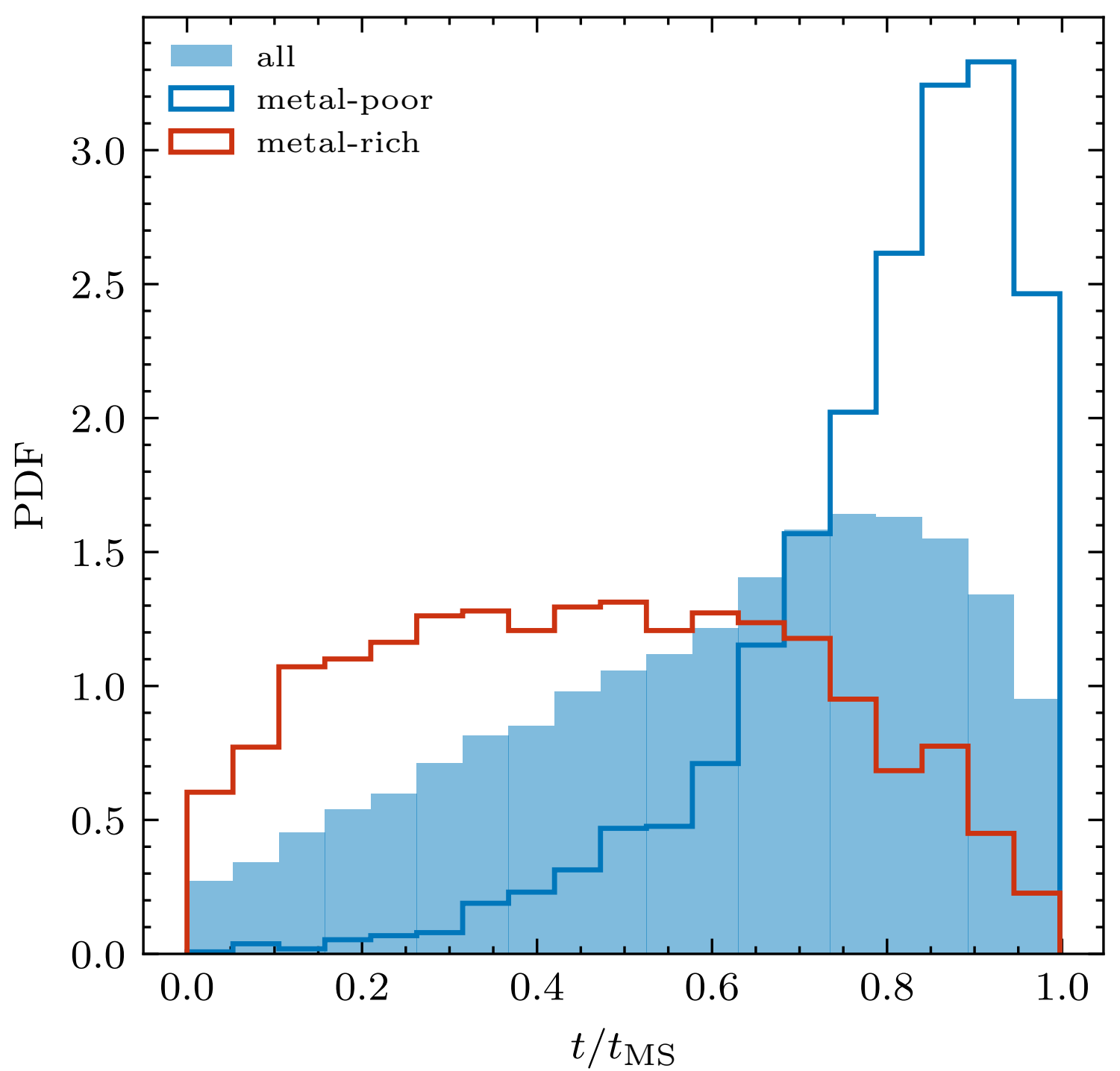

Figure 9. Histograms of the relative ages of (cyan) all normal stars, (blue) the metal-poor subsample $([\mathrm{M} / \mathrm{H}]<-0.2 \mathrm{dex})$, and (red) the metal-rich subsample $([\mathrm{M} / \mathrm{H}]>0.2 \mathrm{dex})$.

\section{CONCLUSIONS}

Using the catalog of Paper I, in this paper we have studied the statistical characteristics of stellar rotation in the MS evolutionary phase. The distribution of the equatorial rotational velocities has been estimated by rectifying the error distribution and correcting for projection effects. We also introduced the ratio $v / v_{\text {crit }}$ to alleviate the dependence of the rotational velocity on mass and age.

Our main results are the following:

- The velocities of the less massive stars $\left(M<2.5 \mathrm{M}_{\odot}\right)$ exhibit a unimodal distribution, with their peak velocity ratio increasing from 0.3 to 0.5 as stellar mass increases.

- There is no clear sign of a lack of slow rotators for $M \sim 1.9 \mathrm{M}_{\odot}$. Even if contamination by $\mathrm{CP}$ stars is better corrected for, the gap is smaller than previously reported.

- A bimodal rotation distribution emerges as stars increase in mass $\left(M>2.5 \mathrm{M}_{\odot}\right)$. The gap between slow and fast rotation becomes prominent for $M>3.0 \mathrm{M}_{\odot}$. Compared with the less massive stars, the rapidly rotating branch exhibits a milder increase with stellar mass and a turnover at $M \sim 3.0 \mathrm{M}_{\odot}$. 
- For stars more massive than $3.6 \mathrm{M}_{\odot}$, more stars reside on the slowly rotating branch compared with stars with masses in the range $2.5 \mathrm{M}_{\odot} \leq M \leq 3.25 \mathrm{M}_{\odot}$.

- Metal-poor $([\mathrm{M} / \mathrm{H}]<-0.2$ dex $)$ stars only exhibit a single branch of slow rotators, with $v / v_{\text {crit }}$ centered at 0.3-0.4, while metal-rich $([\mathrm{M} / \mathrm{H}]>0.2 \mathrm{dex})$ stars clearly show two branches. The difference could be attributed to unexpectedly high spin-down rates in the metal-poor subsample. Strong magnetic fields associated with stars with low metallicity could partly explain the result.

- The bulk of the metal-poor stars are characterized by higher rotation rates than the metal-rich stars.

- Roughly $10 \%$ of CP (Am and mAp) stars exhibit abnormally high rotational velocities.

Our understanding of early-type stellar rotation could be improved in two ways: (1) currently, sample decontamination largely depends on external catalogs, whose homogeneity and completeness are difficult to assess. This caveat is partially caused by the insufficiently high SNR, limited number of observation epochs, and narrow wavelength coverage of our current spectroscopic survey. In the future, armed with more observations collected by LAMOST-MRS, combined with LAMOST-LRS, we can improve the catalog and apply a more consistent decontamination approach. (2) The description of stellar rotation $\left(v / v_{\text {crit }}\right)$ still depends on the stellar evolutionary models adopted. Our sample's homogeneity and size allow us to quantitatively study the rotation distribution as a function of stellar mass, age, and metallicity. For instance, a variate-controlled sample could be used to test the characteristics of stellar evolution on the MS, which in turn could help refine and determine the free parameters remaining in stellar rotation models. Further detailed analysis of the present stellar sample and models of rotating stars will be pursued in forthcoming papers.

\section{ACKNOWLEDGMENTS}

L. D. acknowledges research support from the National Natural Science Foundation of China through grants 11633005, 11473037, and U1631102. The Guoshoujing Telescope (the Large Sky Area Multi-Object Fiber Spectroscopic Telescope; LAMOST) is a National Major Scientific Project built by the Chinese Academy of Sciences. Funding for the project has been provided by the National Development and Reform Commission, China. LAMOST is operated and managed by the National Astronomical Observatories, Chinese Academy of Sciences. This work has made use of data from the European Space Agency (ESA) mission Gaia (https://www.cosmos.esa.int/gaia), processed by the Gaia Data Processing and Analysis Consortium (DPAC; https://www.cosmos.esa.int/web/gaia/dpac/consortium). Funding for the DPAC has been provided by national institutions, in particular the institutions participating in the Gaia Multilateral Agreement.

\section{Facilities: LAMOST}

Software: PARSEC (1.2S; Bressan et al. 2012), Astropy (Astropy Collaboration et al. 2013), Matplotlib (Hunter 2007), TOPCAT (Taylor 2005)

\section{REFERENCES}

Abt, H. A. 1981, ApJS, 45, 437. doi:10.1086/190719

Abt, H. A. 2009, AJ, 138, 28. doi:10.1088/0004-6256/138/1/28

Abt, H. A. \& Morrell, N. I. 1995, ApJS, 99, 135. doi:10.1086/192182

Aerts, C., Mathis, S., \& Rogers, T. M. 2019, ARA\&A, 57, 35. doi:10.1146/annurev-astro-091918-104359

Astropy Collaboration, Robitaille, T. P., Tollerud, E. J., et al. 2013, A\&A, 558, A33.

doi:10.1051/0004-6361/201322068
Attridge, J. M. \& Herbst, W. 1992, ApJL, 398, L61. doi:10.1086/186577

Barnes, S. A. 2003, ApJ, 586, 464. doi:10.1086/367639

Bastian, N., Kamann, S., Amard, L., et al. 2020, MNRAS, 495, 1978. doi:10.1093/mnras/staa1332

Bellm, E. C., Kulkarni, S. R., Barlow, T., et al. 2019, PASP, 131, 068003. doi:10.1088/1538-3873/ab0c2a

Borucki, W. J., Koch, D., Basri, G., et al. 2010, Sci., 327, 977. doi:10.1126/science. 1185402 
Boss, A. P. \& Bodenheimer, P. 1979, ApJ, 234, 289. doi:10.1086/157497

Bouvier, J., Cabrit, S., Fernandez, M., et al. 1993, A\&A, 272, 176

Bressan, A., Marigo, P., Girardi, L., et al. 2012, MNRAS, 427, 127

Buder, S., Sharma, S., Kos, J., et al. 2021, MNRAS, in press. doi:10.1093/mnras/stab1242

Cantat-Gaudin, T. \& Anders, F. 2020, A\&A, 633, A99. doi:10.1051/0004-6361/201936691

Carroll, J. A. 1933, MNRAS, 93, 478. doi:10.1093/mnras/93.7.478

Chen, X., Wang, S., Deng, L., et al. 2018, ApJS, 237, 28. doi:10.3847/1538-4365/aad32b

Chen, X., Wang, S., Deng, L., et al. 2020, ApJS, 249, 18. doi:10.3847/1538-4365/ab9cae

Cui, X.-Q., Zhao, Y.-H., Chu, Y.-Q., et al. 2012, RAA, 12, 1197. doi:10.1088/1674-4527/12/9/003

Dalton, G., Trager, S. C., Abrams, D. C., et al. 2012, Proc. SPIE, 8446, 84460P. doi:10.1117/12.925950

de Mink, S. E., Langer, N., Izzard, R. G., et al. 2013, ApJ, 764, 166. doi:10.1088/0004-637X/764/2/166

Deutsch, A. J. 1967, Magnetic and Related Stars, 181

Donati, J.-F. \& Landstreet, J. D. 2009, ARA\&A, 47, 333. doi:10.1146/annurev-astro-082708-101833

Drake, A. J., Graham, M. J., Djorgovski, S. G., et al. 2014, ApJS, 213, 9. doi:10.1088/0067-0049/213/1/9

Dufton, P. L., Evans, C. J., Hunter, I., et al. 2019, A\&A, 626, A50. doi:10.1051/0004-6361/201935415

Dufton, P. L., Langer, N., Dunstall, P. R., et al. 2013, A\&A, 550, A109. doi:10.1051/0004-6361/201220273

Dunstall, P. R., Dufton, P. L., Sana, H., et al. 2015, A\&A, 580, A93. doi:10.1051/0004-6361/201526192

Ekström, S., Georgy, C., Eggenberger, P., et al. 2012, A\&A, 537, A146. doi:10.1051/0004-6361/201117751

Ekström, S., Meynet, G., Maeder, A., et al. 2008, A\&A, 478, 467. doi:10.1051/0004-6361:20078095

Georgy, C., Ekström, S., Granada, A., et al. 2013, A\&A, 553, A24. doi:10.1051/0004-6361/201220558

Gordon, T. A., Davenport, J. R. A., Angus, R., et al. 2021, ApJ, 913, 70. doi:10.3847/1538-4357/abf63e

Gray, D. F. 1976, The Observation and Analysis of Stellar Photospheres, New York: Wiley Interscience

Guo, Y., Li, J., Xiong, J., et al. 2021, arXiv:2109.09775

Heinze, A. N., Tonry, J. L., Denneau, L., et al. 2018, AJ, 156, 241. doi:10.3847/1538-3881/aae47f

Hou, W., Luo, A., Yang, H., et al. 2015, MNRAS, 449, 1401. doi:10.1093/mnras/stv176

Huang, W. \& Gies, D. R. 2006b, ApJ, 648, 580. doi:10.1086/505782
Huang, W., Gies, D. R., \& McSwain, M. V. 2010, ApJ, 722, 605. doi:10.1088/0004-637X/722/1/605

Huang, Y., Liu, X.-W., Chen, B.-Q., et al. 2018, AJ, 156, 90. doi:10.3847/1538-3881/aacda5

Hunter, I., Lennon, D. J., Dufton, P. L., et al. 2008, A\&A, 479, 541. doi:10.1051/0004-6361:20078511

Hunter, J. D. 2007, Comput. Sci. Eng., 9, 90

Hümmerich, S., Paunzen, E., \& Bernhard, K. 2020, A\&A, 640, A40. doi:10.1051/0004-6361/202037750

Jayasinghe, T., Kochanek, C. S., Stanek, K. Z., et al. 2018, MNRAS, 477, 3145. doi:10.1093/mnras/sty838

Kraft, R. P. 1970, Spectroscopic Astrophysics. An Assessment of the Contributions of Otto Struve, 385

Kunder, A., Kordopatis, G., Steinmetz, M., et al. 2017, AJ, 153, 75. doi:10.3847/1538-3881/153/2/75

Lin, M.-K., Krumholz, M. R., \& Kratter, K. M. 2011, MNRAS, 416, 580. doi:10.1111/j.1365-2966.2011.19074.x

Liu, C., Fu, J., Shi, J., et al. 2020, RAA, submitted; arXiv:2005.07210

Lucy, L. B. 1974, AJ, 79, 745. doi:10.1086/111605

Maeder, A. \& Meynet, G. 2000, ARA\&A, 38, 143. doi:10.1146/annurev.astro.38.1.143

Majewski, S. R., Schiavon, R. P., Frinchaboy, P. M., et al. 2017, AJ, 154, 94. doi:10.3847/1538-3881/aa784d

Matt, S. \& Pudritz, R. E. 2005, ApJL, 632, L135. doi:10.1086/498066

McQuillan, A., Aigrain, S., \& Mazeh, T. 2013, MNRAS, 432, 1203. doi:10.1093/mnras/stt536

Meynet, G., Eggenberger, P., \& Maeder, A. 2011, A\&A, 525, L11. doi:10.1051/0004-6361/201016017

Mouschovias, T. C. \& Morton, S. A. 1985, ApJ, 298, 190. doi:10.1086/163598

Netopil, M., Paunzen, E., Hümmerich, S., et al. 2017, MNRAS, 468, 2745. doi:10.1093/mnras/stx674

Paunzen, E., Hümmerich, S., \& Bernhard, K. 2021, A\&A, 645, A34. doi:10.1051/0004-6361/202038847

Penny, L. R. 1996, ApJ, 463, 737. doi:10.1086/177286

Penny, L. R. \& Gies, D. R. 2009, ApJ, 700, 844. doi:10.1088/0004-637X/700/1/844

Penny, L. R., Sprague, A. J., Seago, G., et al. 2004, ApJ, 617, 1316. doi:10.1086/425573

Prantzos, N., Abia, C., Limongi, M., et al. 2018, MNRAS, 476, 3432. doi:10.1093/mnras/sty316

Preston, G. W. 1974, ARA\&A, 12, 257. doi:10.1146/annurev.aa.12.090174.001353

Pudritz, R. E. 1985, ApJ, 293, 216. doi:10.1086/163227

Qin, L., Luo, A.-L., Hou, W., et al. 2019, ApJS, 242, 13. doi:10.3847/1538-4365/ab17d8

Qin, L., Luo, A.-L., Hou, W., et al. 2021, AJ, in press; arXiv:2105.04741 
Ramírez-Agudelo, O. H., Simón-Díaz, S., Sana, H., et al. 2013, A\&A, 560, A29. doi:10.1051/0004-6361/201321986

Renson, P. \& Manfroid, J. 2009, A\&A, 498, 961. doi:10.1051/0004-6361/200810788

Rivinius, T., Carciofi, A. C., \& Martayan, C. 2013, A\&A Rv, 21, 69. doi:10.1007/s00159-013-0069-0

Royer, F., Gerbaldi, M., Faraggiana, R., et al. 2002a, A\&A, 381, 105. doi:10.1051/0004-6361:20011422

Royer, F., Grenier, S., Baylac, M.-O., et al. 2002b, A\&A, 393, 897. doi:10.1051/0004-6361:20020943

Royer, F., Zorec, J., \& Gómez, A. E. 2007, A\&A, 463, 671. doi:10.1051/0004-6361:20065224

Sana, H. 2017, The Lives and Death-Throes of Massive Stars, 329, 110. doi:10.1017/S1743921317003209

Sana, H., de Koter, A., de Mink, S. E., et al. 2013, A\&A, 550, A107. doi:10.1051/0004-6361/201219621

Sanyal, D., Langer, N., Szécsi, D., et al. 2017, A\&A, 597, A71. doi:10.1051/0004-6361/201629612

Sikora, J., Wade, G. A., Power, J., et al. 2019, MNRAS, 483, 2300. doi:10.1093/mnras/sty3105

Sikora, J., Wade, G. A., \& Rowe, J. 2020, MNRAS, 498, 2456. doi:10.1093/mnras/staa2444
Silverman, B. W. 1986, Monographs on Statistics and Applied Probability, London: Chapman and Hall Skumanich, A. 1972, ApJ, 171, 565. doi:10.1086/151310 Spruit, H. C. 2002, A\&A, 381, 923. doi:10.1051/0004-6361:20011465

Stassun, K. G., Mathieu, R. D., Mazeh, T., et al. 1999, AJ, 117, 2941. doi:10.1086/300881

Sun, W., de Grijs, R., Deng, L., et al. 2019, ApJ, 876, 113. doi:10.3847/1538-4357/ab16e4

Sun, W., Duan, X., Deng, L., et al. 2021, ApJS, submitted; Paper I

Szécsi, D., Langer, N., Yoon, S.-C., et al. 2015, A\&A, 581, A15. doi:10.1051/0004-6361/201526617

Taylor, M. B. 2005, ADASS XIV, 347, 29

Townsend, R. H. D., Owocki, S. P., \& Howarth, I. D. 2004, MNRAS, 350, 189. doi:10.1111/j.1365-2966.2004.07627.x

Yasui, C., Kobayashi, N., Tokunaga, A. T., et al. 2009, ApJ, 705, 54. doi:10.1088/0004-637X/705/1/54

Yasui, C., Kobayashi, N., Tokunaga, A. T., et al. 2010, ApJL, 723, L113. doi:10.1088/2041-8205/723/1/L113

Zorec, J. \&Royer,F. 2012,A\&A, 537, A120. doi:10.1051/0004-6361/201117691 